\title{
Robespierre selon Louis Blanc. Le prophète christique de la Révolution française
}

Jean-François Jacouty

\section{(2) OpenEdition \\ 1 Journals}

Édition électronique

URL : https://journals.openedition.org/ahrf/809

DOI : 10.4000/ahrf.809

ISSN : 1952-403X

\section{Éditeur :}

Armand Colin, Société des études robespierristes

\section{Édition imprimée}

Date de publication : 1 mars 2003

Pagination : 103-125

ISSN : 0003-4436

\section{Référence électronique}

Jean-François Jacouty, « Robespierre selon Louis Blanc. Le prophète christique de la Révolution française ", Annales historiques de la Révolution française [En ligne], 331 | janvier-mars 2003, mis en ligne le 18 avril 2008, consulté le 24 avril 2022. URL : http://journals.openedition.org/ahrf/809 ; DOI : https://doi.org/10.4000/ahrf.809 


\title{
ROBESPIERRE SELON LOUIS BLANC LE PROPHÈTE CHRISTIQUE DE LA RÉVOLUTION FRANÇAISE
}

\author{
JEAN-FRANÇOIS JACOUTY
}

\begin{abstract}
Opposant radical à Louis-Philippe, et bientôt révolutionnaire de 1848 , L. Blanc est partisan d'une République jacobine et socialiste. Son engagement politique s'appuie aussi sur une Histoire de la Révolution française (1847-1862). II distingue 1789 de 1793 par l'opposition de deux principes : individualisme et fraternité. Si le premier a favorisé la bourgeoisie, le second devait affranchir le peuple, opprimé et pauvre, mais sans éliminer la bourgeoisie. Dès 1789, Robespierre aurait été quasiment seul, dans la classe politique, à défendre la perspective d'une France authentiquement fraternelle : politiquement unie dans une démocratie égalitaire, socialement solidaire (et favorable aux pauvres), mais aussi liée par l'amour évangélique. Robespierre est ainsi I'héritier de Jésus et de Vapôtre Rousseau : sa parole de vérité en fait un vrai prophète. Défenseur de l'Humanité et d'un Droit de Justice, il est populaire auprès des masses et influent aux Jacobins, mais reste politiquement isolé. Même en 1793 il demeure prophète : conscience et guide de la Révolution, il en impose toujours par son verbe, mais le pouvoir lui échappe. N'étant pas le dictateur dénoncé par ses ennemis, il ne peut accomplir la Révolution et empêcher les violences terroristes, si contraires au Droit. Le 9 thermidor marque aussi la fin de la Révolution. L. Blanc y voit une tragédie sacrificielle : la Passion d'un nouveau Christ. Chargeant Robespierre de leurs crimes et d'un despotisme imaginaire, les révolutionnaires renoncent en fait à la Révolution, dont I'Incorruptible incarnait au plus haut l'Idée: Mais le prophète lègue son message aux générations du XIXe siècle, à charge de l'accomplir.
\end{abstract}

Mots clés : Grand Homme ; République (démocratique et socialiste) ; Peuple; Bourgeoisie ; Humanité ; Jésus ; Prophète.

Comment apprécier le rôle des grands hommes dans le processus historique ? Aux héros de l'histoire et de la culture classiques, marquées par l'héritage antique, les Lumières ont opposé la supériorité des grands hommes, même si elles mettent aussi l'accent sur les structures politiques et 
les évolutions profondes de la société et de l'économie (1). Au début du XIXe siècle, où le héros est de retour avec le romantisme, historiens et philosophes tiennent toujours grands hommes et hommes supérieurs pour les agents majeurs de l'Histoire (2). Mais la construction de leur image résulte autant d'un héritage de faits, dont ils ont été responsables, que de sa lecture idéologique. Donc d'une approche du passé qu'une vision du monde - un système de principes et de valeurs - interprète, en termes finalistes, à travers les enjeux du présent et les attentes d'un futur. Reconnaître une individualité supérieure signifie donc que son action et sa pensée cautionnent un sens de l'Histoire. Mais dans ces échanges - constants et complexes - entre passé et présent que de jugements contradictoires lorsque sa légitimité est discutée. Au point que la destinée posthume d'un grand homme peut peser plus que sa vie réelle, ce qui brouille son statut historique.

Il en est ainsi de Robespierre, personnalité au rôle reconnu mais qui reste le responsable politique le plus controversé de la Révolution française car il fut au cœur de l'épisode - très discuté - de 1793. A-t-il été, ou non, un grand homme? Fut-il tyran, selon le jugement - largement partagé - des Thermidoriens, et des historiens républicains J. Michelet et E. Quinet (3) ? Ou bien saint pour les socialistes et les républicains radicaux qui ont rêvé, contre la monarchie de Juillet, de ressusciter la République jacobine (4) ? Ces approches contraires révèlent les tensions - historiques et politiques de l'opposition au régime de Louis-Philippe, alors même que les événements de 1830 (nouveau 1789?) ont semblé relancer la Révolution. Aux côtés de Ph. Buonarotti, Buchez et Roux, A. Laponneraye, Ét. Cabet, A. Esquiros, L. Blanc illustre une historiographie radicale qui opère un basculement de 1789 à 1793. Préparant les voies de l'avenir, elle exalte la République jacobine dans un sens socialiste et chrétien, voire mystique, et réhabilite la figure de Robespierre (5). Apportant une importante contribu-

(1) Aux héros classiques, princes guerriers et conquérants, les Lumières opposent les grands hommes, législateurs, intellectuels et éducateurs qui instituent, eux, l'Humanité. Voltaire souligne toute leur importance (cf. son Essai sur les mours, 1756). Mais il a aussi fixé le programme d'une nouvelle histoire sous l'influence de l'économie politique et des sciences morales et politiques, alors en plein essor (cf. son article « Histoire » de l'Encyclopédie).

(2) Au début du XIXe siècle, Hegel, Guizot, V. Cousin... soulignent l'importance des grands hommes, à la fois individualités et expressions d'une totalité historique dont ils incarnent et assument les tendances dominantes. Mais A. Thierry réagit à cette tendance en faisant ressortir l'autonomie et le rôle des masses.

(3) MICHELET et E. QUINET sont, respectivement, auteurs d'une Histoire de la Révolution française (1847-1853) et de La Révolution (1854, réed. 1865).

(4) Cf., par exemple, le témoignage du peintre Degas. Visitant la veuve du jacobin Lebas, sa mère s'étonne de voir accrochés les portraits de Robespierre, Saint-Just et Couthon : « - Quoi vous gardez encore ici le visage de ces monstres !» «- Tais-toi, réplique avec ardeur MmeLebas, c'étaient des saints ! » (rapporté par Paul VALÉRY dans Variété IV, 1938, p. 129).

(5) Après l'œuvre-phare de Philippe BUONAROTT, La Conspiration pour l'Égalité dite de Babeuf (1828, Bruxelles/1830, Paris), BUCHEZ et Roux compilent (et commentent) une monumentale Histoire parlementaire de la Révolution française (1834-1840), Albert LAPONNERAYE édite les Euvres de Maximilien Robespierre (1840) après avoir écrit une Histoire de la Révolution française (1838), Étienne CABET donne une Histoire populaire de la Révolution française (1838) et Alphonse ESQUIROS son Histoire des Montagnards (1847). 
tion dans ce sens, L. Blanc revêt l'homme politique de la dimension supérieure d'un prophète, voire d'un apôtre, posant ainsi le problème de sa responsabilité et de son rôle réels dans les tragiques événements de l'an II. Pourtant «homme du peuple» (P. Viallaneix), Michelet pourfend vigoureusement ce courant dans son Histoire de la Révolution française. Républicain et démocrate, il nie le caractère chrétien et socialiste de la Révolution, et dénonce la tyrannie jacobine dont le peuple - le héros de 1789, qu'il préfère à 1793 - a été la victime. L. Blanc se charge de lui répliquer, pour le critiquer largement, dans sa propre Histoire de la Révolution française, contemporaine de l'œuvre de Michelet.

\section{Ombres et lumière de la Révolution : Robespierre dans le mystère de l'Histoire}

Intellectuel et militant de la cause socialiste, L. Blanc a écrit les douze volumes de son Histoire de la Révolution française de 1847 à 1862 (6). Dans cette œuvre historienne et politique, il a cherché à synthétiser République jacobine et socialisme. Autrement dit, féconder par la démocratie politique de 1793 la démocratie sociale à venir (objectif conçu sous la monarchie de Juillet et maintenu malgré l'échec de 1848). Attentif à la misère du peuple et critique pour l'égoïsme de la bourgeoisie, L. Blanc souhaite néanmoins les rassembler dans un socialisme d'union des classes (7). Cet objectif - hautement moral - d'une Humanité réconciliée et épanouie, monde de liberté et de fraternité, fin même de l'Histoire, révèle une conception idéaliste de celle-ci. Reposant sur un spiritualisme philosophique et religieux, caractéristique de l'époque, elle recourt largement aux idées et aux principes pour expliquer la dynamique de l'Histoire. Or la Révolution française est une étape clé dans le processus qui fait émerger la nouvelle Humanité. Pourtant, son déroulement a été proprement dramatique, outre qu'elle s'est - provisoirement - arrêtée en Thermidor. Par ailleurs, L. Blanc fait ressortir combien les enjeux et les luttes de la Révolution ont de plus en plus conduit ses acteurs à se mobiliser et à s'investir chaque jour davantage. Or, à partir de 1793, surtout, les difficultés croissantes et les crises ont fait ressortir toutes

(6) L'Histoire de la Révolution française de Jules MICHELET paraît de 1847 à 1853. Comme lui, L. Blanc commence donc à publier son oeuvre à la veille de la Révolution de Février. Il interrompt son travail en devenant alors membre du gouvernement provisoire et président de la Commission du gouvernement pour les travailleurs (Luxembourg). Il le reprendra pour l'achever à Londres, où il s'exile, après les événements du 15 mai 1848 (qui marquent l'échec des révolutionnaires de Février). Sur ce travail, cf. notre communication sur « Louis Blanc et la construction de l'Histoire », colloque Louis Blanc, le Socialisme et la République, Université Paris X- Nanterre, février 2000.

(7) L. Blanc souhaite une « intime association de tous les intérêts » dans un esprit de fraternité évangélique, (cf. ci-après), Histoire de la Révolution française, HRF, Deux Révolutions, Livre (L.) XIII, chap. 4. 
les contradictions des hommes. Aussi les événements ont-ils de plus en plus exacerbé passions et violences, charriant le bien et le mal, les sentiments les plus élevés comme les instincts les plus bas, jusqu'aux pires crimes (8). Selon une approche romantique, L. Blanc montre la mort côtoyant de plus en plus la vie, et les ténèbres la lumière. Il ne cherche donc pas à relativiser, voire à nier, les dérives et les crimes de ce qu'il tient pour une seconde Révolution, puisqu'elles en font partie. D'où sa préoccupation majeure de légitimer 1793, l'essence même de la Révolution, en le dissociant de sa part maudite (mais pourquoi le mal a-t-il, finalement, pesé autant que le bien dans une cause si juste?).

Dans un processus aussi complexe, L. Blanc assigne aux grands hommes un rôle déterminant : dans le drame révolutionnaire, largement marqué par la fatalité (ou logique de l'histoire), ils ont - momentanément - incarné une idée et une ligne politiques. C'est ainsi que Mirabeau et Robespierre ont représenté ce qu'il tient pour les deux principes successifs de la Révolution : l'individualisme de 1789, puis la fraternité de 1793. Mais en consacrant celle-ci comme son principe même, sa vérité profonde, Robespierre en incarne, à ses yeux, tout l'esprit. Mais d'avoir été au cœur de 1793 il a vécu le paroxysme des contradictions et des dérives de la Révolution, alors même qu'il en exaltait tout l'idéal progressiste. Mais que sa destinée se soit terminée tragiquement signifie-t-il que le mal l'aurait emporté sur le bien ? Sinon, comment expliquer cette destinée pour en sauvegarder, surtout à l'intention des générations futures, toute la force politique (et prophétique) ?

Puisque contrastes et contradictions sont inhérents au drame, le problème historique de L. Blanc est de comprendre ceux de la Révolution. Or le christianisme qui, avant 1848, imprègne tant son historiographie républicaine et socialiste, va l'y aider. Christianisme démocratique s'entend - celui des origines - il n'est pas seulement associé à l'idéal révolutionnaire pour avoir aussi prôné l'égalité. Le premier XIXe siècle - marqué par un romantisme très spiritualiste - intègre également sa dimension proprement religieuse. Or, à défaut d'une approche proprement dialectique, le christianisme permet d'expliquer les contraires, exigence particulière lorsque le bien est en question. C'est qu'étant au plus près du mystère des faits et des hommes, il permet de mieux comprendre le mal comme l'épreuve de la vérité, qui finit toujours, l'Histoire le prouve, par en triompher (9). En relativisant la face sombre de la Révolution, cette approche postule son achèvement puisqu'elle en sauvegarde, en définitive, l'idéal et les principes fondateurs. Que le mal ait effectivement coexisté

(8) La Révolution, écrit L. Blanc, fut un «drame immense », un «chaos de sentiments contradictoires », (Id., L. VII, chap. 9 et L. X, chap. 7).

(9) «Mais à côté du bien pourquoi le mal?, s'interroge L. Blanc, demandez à Dieu», (Id., L. V, chap. 8). Et de souligner que « le cœur de l'homme contient le ciel et l'enfer», (L. VIII, chap. 2). 
avec le bien ne remet donc pas en question la légitimité de la Révolution, réplique L. Blanc à ceux qui la combattent. Or cette contradiction a particulièrement caractérisé 1793. Il peut même arriver, dans de tels contextes, que les grands hommes - éclaireurs des consciences et guides de leur peuple - soient persécutés jusqu'au sacrifice de leur vie, à l'exemple de Jésus lui-même (dont ils partagent la mort rédemptrice). C'est pourquoi des circonstances historiques contraires, l'aveuglement et les imperfections des hommes, peuvent les transformer en prophètes. La raison essentielle est cette distance entre leur parole de vérité, en attente d'être accomplie, et une réalité sociale et morale qui s'y oppose encore.

À L. Blanc - spiritualiste religieux personnellement panthéiste, mais éduqué dans l'ancienne religion (10) - le christianisme a fourni des clés - principes, analogies et images - pour mieux penser Robespierre dans la cohérence, l'exemplarité et la postérité de sa destinée brisée. Usant de rhétorique religieuse pour camper l'Incorruptible en prophète christique, L. Blanc a voulu signifier que la vie finit par l'emporter toujours sur la mort, comme le bien sur le mal. À charge pour les générations futures - celles du XIXe siècle - de ressusciter l'esprit de 1793, qu'il a douloureusement incarné au prix de son sang, pour en réaliser - enfin - la promesse. «Seul le bien est absolu, seul il est nécessaire [écrit-il], le mal dans le monde est un immense accident» (11).

\section{Généalogie de Robespierre : disciple de Jean-Jacques et de Jésus}

Dans une longue analyse des Origines et causes de la Révolution (12), L. Blanc explique le processus historique, qui l'a engendrée, par l'action de trois grands principes : autorité, individualisme et fraternité. Incarné par l'Église catholique, puis l'État absolutiste, le principe d'autorité a été de plus en plus contesté au XVIIIe siècle. En particulier par le principe d'individualisme, devenu dominant et qui le reste au $\mathrm{XIX}^{\mathrm{e}}$ siècle. D'origine protestante, il exalte l'autonomie de l'individu. À une bourgeoisie en plein essor, il apporte tous les éléments de son règne (le laisser-faire) : Voltaire, le grand homme du siècle, défend ainsi le droit individuel, Montesquieu l'État libéral, les économistes la liberté de production et d'échange. Mais ce principe en isolant l'individu du reste de la société, objecte L. Blanc, ne peut que

(10) L. Blanc, issu d'un milieu bourgeois déclassé et légitimiste, victime des révolutions de 1789 et 1830, a étudié au collège jésuite de Rodez grâce à une bourse de Louis XVIII. Il évolue vers la République et le socialisme après les Trois Glorieuses. (Cf. ses biographies par Ed. RENOUARD, 1928, et J. VIDALENC, 1948). Michelet lui reprochera vivement son christianisme (cf. de son Histoire de la Révolution française, la préface de 1868).

(11) HRF, Origines et causes de la Révolution française, conclusion (tome 1).

(12) $I d$. 
profiter aux plus forts : favorisant, dès lors, l'anarchie sociale, il prépare l'oppression du peuple par la bourgeoisie (13).

C'est pourquoi une minorité lui oppose le principe de fraternité. Défendu autrefois par des chrétiens dissidents (Vaudois, Anabaptistes, Hussites, Frères Moraves), il est devenu, maintenant, la philosophie de Jean-Jacques Rousseau. À un Voltaire triomphant et ami des puissants, L. Blanc oppose l'étonnante figure d'un Jean-Jacques pauvre et isolé : dans le monde riche et brillant des Lumières, il prêche contre tous une vérité trop oubliée (14). Apôtre et bon prêtre, inspiré par la sainteté de l'Évangile et l'exemple du Nazaréen, selon les propres termes de L. Blanc, son déisme est en fait le vrai christianisme. Aussi oppose-t-il au rationalisme dominant - condamné comme réducteur et diviseur - un tout autre message. À l'individualisme anarchique et égoïste, qu'il favorise, il veut substituer la force du sentiment, la puissance d'aimer de Jésus. Seul, il peut fonder une société unie, solidaire et fraternelle, l'idéal de l'Humanité étant d'être constituée dans une vraie communauté sociale, sans oppositions de classes (15). Approche que L. Blanc juge autant chrétienne que socialiste : pour critiquer l'hégémonie bourgeoise de l'époque, il cherche après Rousseau, tel qu'il l'interprète, à unir, dans une même démarche, le message religieux d'amour et d'égalité à la perspective d'union, sinon d'abolition, des classes. Se réclamant à plusieurs reprises de l'Évangile, L. Blanc n'envisage pas l'émancipation sociale sans que l'amour fraternel entre les hommes vienne la consolider, finalité éminemment divine (16). Il peut écrire que «le progrès consiste à réaliser de plus en plus au sein des sociétés humaines la loi d'union qui se voit aux œuvres divines » (17).

Plus encore, le Jean-Jacques de L. Blanc serait «une grande âme déchirée », voire le «martyr de sa propre gloire ». Il le campe ainsi en Christ souffrant, s'adressant au peuple également souffrant (les «damnés de la terre »). Mais même si les pauvres - avant-garde de l'Humanité pareille aux premiers disciples de Jésus - sont encore les seuls à écouter son message, celui-ci commence à se répandre à la veille de la Révolution. À Rousseau L. Blanc rattache d'autres courants de pensée, ainsi les philosophes

(13) L. Blanc décrit simplement les classes. Les bourgeois sont les «citoyens qui possèdent des instruments de travail ou un capital, et travaillent avec des ressources propres». Quant au peuple, ou prolétaires, il est formé de « citoyens qui ne possédant aucun capital dépendent d'autrui complètement » (cf. HRF, Origines et causes de la Révolution française : plan, L. II, et Deux révolutions, L. VI, chap. 1).

(14) Id, L. III, chap. 1.

(15) Si L. Blanc montre l'époque moderne dominée par le conflit entre bourgeoisie et peuple, il s'y attarde peu. De fait il ne le décrit pas comme une lutte de classes, terme qu'il n'emploie jamais parce qu'il contredit, au fond, son projet socialiste.

(16) L'Évangile, déclare L. Blanc, « législation sublime de l'égalité » et « code du monde affranchi », inspire «un amour auguste et profond entre les hommes et les classes» (HRF, Deux révolutions, L. V, chap. 6 et L. VIII, chap. 5).

(17) De la solidarité qui en découle « le premier anneau tient au trône de Dieu », conclut L. Blanc (M,L. L. I,chap. 14). 
illuministes, comme $\mathrm{Cl}$. Fauchet, qui en sont effectivement influencés : eux aussi, se réclamant de Jésus, exaltent amour fraternel et union des cœurs contre l'égoïsme des individus, l'exploitation de l'homme et la division sociale. Tous préparent une autre Révolution.

L'important est que Jean-Jacques soit le maître de Robespierre : la politique que celui-ci défendra est issue - principalement - de sa pensée. Il lui lègue sa religion de justice, de fraternité et d'harmonie sociales, mais attentive au peuple des pauvres, religion que Jésus a été le premier à formuler (18). D'ores et déjà le XVIII siècle est traversé par deux courants de pensée. La division intellectuelle des Lumières, dont l'origine est religieuse, engendrera les deux révolutions décrites par L. Blanc, 1789 puis 1793. L'ascendance de Robespierre serait donc rousseauiste et chrétienne, mais de filiation plutôt catholique (le milieu d'origine de L. Blanc). Le principe de fraternité que défend Rousseau s'oppose au principe d'individualisme. Voltaire en est le principal théoricien, mais son ascendance religieuse est protestante. Tout en prêchant l'union sociale, L. Blanc n'est pas moins marqué par la césure des deux grandes religions, à l'origine, donc, de deux cultures politiques antagonistes. Il situe ainsi Rousseau et Robespierre, même déistes, dans la tradition catholique, leur socialisme - ou présocialisme- s'inscrivant dans son idéal communautaire. Courant très influencé par Lammenais dont les Paroles d'un croyant (1834) développent un prophétisme démocratique et socialiste (« le cri du pauvre [déplore-t-il] monte jusqu'à Dieu, mais il n'arrive pas jusqu'à l'oreille de l'homme »). Cette filiation catholique permet à L. Blanc, comme à Laponneraye, d'accuser les traits christiques de Jean-Jacques puis Robespierre (Christs souffrants et rédempteurs). Michelet ne pouvait accepter cette immersion de la Révolution dans le christianisme, car elle le dépasse en étant elle-même religion (voire Église) (19).

Toujours attentif aux signes du destin, selon le romantisme du temps, L. Blanc est frappé par la mort simultanée des deux grands philosophes : la disparition de «ces deux grands flambeaux, leur double mort est une des plus imposantes, car elle détermine le moment précis où la pensée se fit homme et où les deux doctrines rivales devinrent deux révolutions » (20).

(18) Ce Jésus sera celui de 1848 : le «prolétaire de Nazareth ». Son image a été forgée tant par l'historiographie radicale de la Révolution que par le romantisme social de l'époque, tous deux très religieux. Laponneraye proclame ainsi « Jésus, Rousseau, Robespierre, trinité sainte et sublime qui résume en elle les principes d'égalité et de fraternité, qui luit comme un rayon rédempteur au front de l'Humanité asservie » (Euvres de M. Robespierre, Notice historique, t. I, p. 5). Esquiros, déjà auteur d'un Évangile du Peuple (1840), écrit que la Montagne, incarnation même de la Révolution, a été « le bras de Dieu, l'Évangile armé » (Histoire des Montagnards, $t$.1, Introduction).

(19) Rappelons l'importance de l'interprétation protestante de la Révolution, en liaison avec le libéralisme : en témoignent particulièrement $\mathbf{M}^{\mathrm{me}}$ de Staël et $\mathrm{B}$. Constant, Guizot et E. Quinet. Ils contrastent aussi avec le jacobin Buchez, partisan d'un socialisme catholique. Hostile aux interprétations chrétiennes de la Révolution, Michelet s'en prend ainsi vivement à L. Blanc, ce « demi-chrétien à la façon de Rousseau et Robespierre » dont il dénonce le «papisme socialiste» (cf. sa préface de 1868, op. cit.).

(20) $H R F$, Deux révolutions, introduction du L. I. 


\section{Prodromes du destin (1789-1792)}

Dans l'Histoire de la Révolution française, les apparitions de Robespierre, selon une approche romantique, restent nimbées de mystère. Il apparaît, pour la première fois, lors de la procession des députés aux États généraux à Versailles, le 5 juin 1789 : L. Blanc le campe, d'emblée, en homme du destin puisque, prévient-t-il, «dans le drame du temps chacun a son rôle tracé d'avance par le grand auteur mystérieux » (21). Alors que les autres élus manifestent autant d'enthousiasme que de perplexité sur la suite des événements, «un seul dans ce cortège, un seul pressentait alors, illuminé qu'il était par sa conviction, les conséquences suprêmes. Or celui-là se trouvait parmi les plus obscurs, il marchait retiré en lui » (22).

Pour quelque temps Robespierre, nouveau Jean-Jacques, n'est donc qu'un obscur député, mais ses premières interventions ont vite fait de l'imposer. Une mystérieuse aura baigne le personnage, augurant de sa destinée exceptionnelle et préfigurant le prophète qu'il sera bientôt, puisque lui seul saurait pressentir les événements et dire leur vérité (23). Mais il sera un prophète laïc, parlant et agissant dans les débats, et selon les procédures, d'une vie politique naissante. De son activité comme député à l'Assemblée constituante, puis principal animateur du Club des Jacobins sous l'Assemblée législative, L. Blanc fait ressortir deux faits essentiels.

D'abord, à l'occasion des grands débats politico-intellectuels, Robespierre aurait été le seul à défendre le principe de fraternité contre le principe d'individualisme, largement dominant. Or celui-ci divise la nation en assurant la domination de la bourgeoisie sur le peuple grâce au laisser-faire. Décrivant une situation politique binaire, L. Blanc oppose ainsi Robespierre à tous les héritiers de Voltaire et Montesquieu : Monarchiens et Constitutionnels (dont Sieyès) de l'Assemblée constituante, puis Feuillants de l'Assemblée législative. À tous il reproche, surtout, d'avoir divisé les citoyens en actifs et passifs, critique majeure car cette coupure sanctionne politiquement la fracture sociale. L. Blanc n'hésite même pas à ranger les Girondins, pourtant plus rousseauistes que voltairiens, dans le camp adverse : ces bourgeois démocrates, décrits comme intellectuels et « artistes égarés en politique », seraient aussi favorables à l'individualisme (24). Selon L. Blanc, Robespierre est le seul défenseur de la pensée de Rousseau, l'apôtre des Lumières. Au rationalisme réducteur il oppose toujours la force du sentiment : alors que le premier isole les hommes et les divise en classes antagonistes, le second, bien au contraire, les rassemble dans la solidarité et

(21) Id., L. V, chap. 8.

(22) Id., L. I, chap. 8.

(23) Id., cf. L. II, chap. 3 et 4.

(24) Id., cf. L. VII ; chap. 2. Approche sans doute influencée par l'Histoire des Girondins (1847) de LAMARTINE. 
un bonheur partagé. L'Humanité demeure donc l'idéal à construire, et même si Robespierre ne conteste pas le «droit des individus », ces derniers, précise-t-il, ne peuvent se déterminer que «d'après leurs rapports avec l'avantage de tous»(25). Dans cette perspective, la religion civile de Rousseau doit jouer un rôle essentiel : garantissant « les dogmes relatifs à l'accomplissement des devoirs sociaux et des vertus civiques »(26), elle enseigne, d'abord, l'amour de l'Humanité et particulièrement du peuple (27). Prophète, même laïc, Robespierre l'est d'autant plus que son idéal a aussi une dimension religieuse (aspect capital pour L. Blanc, qui mêle déisme et christianisme, comme chez Rousseau).

D'autre part, Robespierre impose son autorité au Club des Jacobins, qu'il contrôle, selon L. Blanc, depuis mai 1790 (lorsque les modérés partent fonder le Club de 1789). Il en fait une «société avant tout politique », « une armée » même, solidement organisée par le club parisien fédérant les filiales provinciales (28). Or l'objectif principal de ce club, insiste L. Blanc, est de défendre le peuple (plebs) dont Robespierre aurait toujours eu l'instinct, au point d'être vénéré par lui jusqu'à sa mort. Dans le monde politique bourgeois des Assemblées révolutionnaires, il paraît donc isolé. Mais, précise L. Blanc, défendre le peuple ne signifie pas sacrifier les nécessités supérieures de l'ordre nouveau. Si la fraternité conduit à se pencher sur un peuple momentanément victime de mauvaises conditions sociales, son idéal est bien de concilier toutes les classes dans le peuple politique (populus), en les y mêlant dans une parfaite égalité fraternelle (loin de toute lutte de classes). L'unité politique, garantie par le suffrage universel, est le fondement de l'union sociale (29). Soucieux de défendre pauvres et défavorisés - la meilleure part, sans doute, du peuple politique - Robespierre n'est pas moins attaché à la construction d'un ordre garantissant à tous la justice. Mais chez un prophète, outre son isolement, il y a toujours une distanciation, sa parole supérieure paraissant comme lointaine. C'est ainsi que L. Blanc nous montre, pour le critiquer à demi, un Robespierre réservé et froid, « incarnation glacée d'un principe, statue du Droit» (30). Refusant ainsi de

(25) Id., cf. L. I, chap.14 (pour les Constitutionnels) et L. VII, chap. 9 (pour les Girondins).

(26) Id, L. IV, chap. 12.

(27) Dieu, déclare L. Blanc, est « complice du peuple, protecteur des faibles et des opprimés » (id, L. VII, chap. 9).

(28) Ibid., L. V, chap. 5. Si ce type d'organisation, selon le jacobin L. Blanc, est nécessaire pour assurer le succès politique du peuple, il fonde aussi une dictature lui objecte Michelet (cf. son Histoire de la Révolution française).

(29) Le peuple selon Rousseau, que reprend Robespierre, est « la généralité des citoyens dont la société se compose » (cf. le Contrat Social,). Leur conception, et celle de L. Blanc, est proche de la pensée de Michelet, toujours critique envers les approches bourgeoises et socialistes qu'il accuse d'aviver les oppositions de classes. Chez lui le peuple-plebs, d'où est issue la bourgeoisie, incarne le mieux le peuple-populus (cf. Paul VIALLANEIX, La voix royale-Essai sur l'idée de peuple dans l'œuvre de Michelet, Paris 1971, Livre III, chap. I).

(30) Op. cit., L. VI, chap. 1. Ce Robespierre est loin de l'effusion romantique de 1848. 
«faire peuple» (31), il apparaît en quelque sorte déshumanisé, comme réduit à une Idée (ce que renforce encore sa réputation d'Incorruptible). Mais sa parole agit toujours magiquement sur ses auditeurs, éclat d'une vérité qu'ils révèrent, ou attendent plus ou moins consciemment, même si beaucoup n'en comprennent pas encore le sens.

À la fois «penseur, philosophe, grand homme d'État» (32), Robespierre a des qualités qui l'élèvent au-dessus des autres révolutionnaires. Mais son objectif, L. Blanc y reviendra souvent, est d'abord politique : au nom de l'égalité, il faut d'abord constituer le peuple souverain, socle nécessaire du principe defraternité et condition préalable à tout changement. Cet objectif -jacobin est aussi celui de L. Blanc et des autres socialistes politiques du XIXe siècle (tous attachés à l'héritage de 1793, première étape de la construction du socialisme). Il reste pourtant que la réponse aux problèmes du peuple ne saurait être seulement politique. Même si les circonstances historiques privilégient l'action politique, l'intégrer dans le peuple politique résoudra-t-il les difficultés provoquées par son infériorité économique et sociale (exploitation du travail, misère, chômage et faim) ? Là est la difficulté à promouvoir Robespierre en véritable socialiste, comme le remarquera Michelet (33). C'est pourquoi L. Blanc donne une grande importance au Cercle Social de $\mathrm{Cl}$. Fauchet et Bonneville. Appartenant, on l'a vu, au courant illuministe et se rattachant autant à l'Évangile qu'à Rousseau, il préconise le «principe d'association», une «intuition d'avenir » («seul moyen [commente L. Blanc] d'appeler chaque membre de la famille nationale à la jouissance du droit de propriété ») (34). Robespierre et Cl. Fauchet auraient donc pensé les éléments complémentaires de la République socialiste du XIX ${ }^{\mathrm{e}}$ siècle, terme logique de la Révolution.

L'ascendant croissant de Robespierre et l'accélération des événements vont peu à peu le propulser au premier rang. Son heure sonne symboliquement lorsque meurt Mirabeau, dont la corruption contraste tant avec sa vertu. Cette mort annonce le déclin du principe d'individualisme qu'il a longtemps incarné, mais aussi compromis par ses errements personnels. Voyant dans son décès le signe symbolique d'un destin toujours mystérieux, donc divin, L. Blanc, non sans emphase, écrit : «Quand l'acteur s'en va, c'est qu'il n'avait plus rien à faire sur la scène, et la preuve que son rôle est fini c'est sa mort » (35).

(31) Robespierre refuse ainsi de sacrifier au sans-culottisme des Girondins (id., L. VII, chap. 7). Par ailleurs, L. Blanc critique les révolutionnaires anarchiques des Cordeliers (eux aussi disciples de Voltaire !) : « révolutionnaires sans freins, mystiques de l'anarchie », bientôt athées, ils défendraient « la Révolution pour tous et chacun pour soi », principe opposé à celui des Jacobins («chacun pour la Révolution et la Révolution pour tous») (cf. id., L. V, chap. 5).

(32) M., L. VIII, chap. 1.

(33) Cf. toujours la préface de 1868 de MICHELET, op. cit. Mais L. Blanc aborde peu l'œuvre proprement sociale et économique des Montagnards, sauf la loi du Maximum dans un chapitre isolé (il en montre les effets positifs puisqu'elle suppose « une intime association de tous les intérêts », id., L. XIII, chap. 4).

(34) Id., L. V, chap. 5 (L. Blanc ne remet donc pas en cause le droit de propriété).

(35) M, L. V.chap. 8. 


\section{Prophète ou homme de pouvoir? (1793-1794)}

La Révolution démocratique du 10 août 1792, fondée sur l'union de la bourgeoisie et du peuple, devait, logiquement, assurer la victoire de Robespierre. La Révolution étant, avant tout, «une idée, un principe [écrit L. Blanc], à celui qui représentait cette idée avec le plus d'élévation morale, et servit ce principe avec le plus de rectitude, à celui-là resta la force » (36). Mais ce serait encore oublier que l'Histoire ne se réduit pas à la seule lutte des idées et des principes, puisque les passions et les sentiments contrastés des hommes, que les révolutions éveillent, viennent souvent en contrarier le déroulement. C'est ainsi qu'aux côtés de Robespierre, incarnation toujours froide de l'Idée (37), Danton, que préfère Michelet, exprime alors toute la vitalité d'hommes dont les «passions brûlantes » charrient - inévitablement le bien comme le mal, les vices et les vertus. Mais cette - symbolique - dualité, en confirmant la séparation de la prophétie d'avec la politique, risque - encore- de limiter Robespierre au magistère de la parole. Preuve d'un inachèvement virtuel de la Révolution que l'imperfection des hommes empêche d'accomplir, leçon toujours à méditer pour les générations futures.

Dans ces conditions, quels furent le rôle et l'influence réels de Robespierre : tant à l'époque de la rivalité entre Girondins et Montagnards, qu'après le triomphe de ceux-ci ?

Dans le conflit politique entre Girondins et Montagnards, au début de la Convention, L. Blanc nous montre Robespierre défendant farouchement l'unité des républicains. Puisque les deux partis montrent un même attachement au nouveau régime, leur lutte implacable affaiblit inutilement la Révolution. À l'entraînement dangereux des passions il oppose une nécessité supérieure, celle de l'union au nom du Droit dont il incarne toujours l'Idée (38). Prophète il demeure, en restant au-dessus des passions. Pourtant, même si les procédures régulières que le Droit aurait dû inspirer ont manqué pour canaliser les affrontements politiques, la lutte des deux principes contraires de la Révolution ne continue pas moins dans le nouveau cadre de la République. De fait, les Girondins, que L. Blanc décrit toujours aussi sommairement comme voltairiens, défendent l' individualisme dont la seule bourgeoisie - qu'ils représentent prioritairement - tire profit. Ils font face à Robespierre, le principal et plus authentique représentant de la

(36)Id, L. VIII, chap. 1.

(37) Robespierre est l'«être abstrait de la Révolution» (id., chap. 7). Pour Laponneraye, il a été « révolutionnaire par conviction et par principes » (Mémoires de M. Robespierre, $t .1, \mathrm{p} .12$ ). Même appréciation chez Michelet qui le considère comme «l'homme des principes », alors que Danton, un authentique homme du peuple, serait la « voix même de la révolution et de la France » (Histoire de la Révolution française, Livre IV, chap. 5).

(38) Cette « lutte des passions, lutte funeste, insensée, horrible, fut le suicide de la Révolution française », comme le déplore L. Blanc (op. cit., chap. 4). De la nécessité de ne pas exagérer les conflits, surtout s'il y a consensus sur l'essentiel, les républicains de 1848 tenteront de tirer la leçon. 
Montagne, défenseur d'une vraie politique de justice. Appuyé par la Commune, il bénéficie toujours de la confiance du peuple de Paris, déclarée ville sainte de la cause révolutionnaire et avant-garde du peuple français (le peuple messianique qui, bientôt, fera « au prix de son sang, versé à flot, la besogne du genre humain ») (39).

Toujours maître du Club des Jacobins, Robespierre forge et propage son programme d'«affranchissement moral, intellectuel et social de la masse du peuple», qui est aussi un programme d'ordre et la finalité même de la Révolution (40). Dans ces conditions, les débats sur la Constitution de 1793 et sa Déclaration des droits de l'homme et du citoyen confrontent des principes antagonistes. Même si tous se réclament de la Souveraineté du Peuple de la République, son concept est aussi social. Robespierre dépose ainsi un projet contre celui de Condorcet. À l'individualisme et au pur rationalisme des Girondins, qui fondent garanties et libertés, conquêtes incontestables, L. Blanc nous le montre opposant toujours à ses adversaires le message de Jean-Jacques : puisqu'il faut construire, avant tout, une société de justice, de solidarité et de réelle égalité, seuls le sentiment et l'esprit de fraternité, qui rassemblent au lieu de diviser, accompliront le véritable objectif de la Révolution. Robespierre corrige ainsi les Droits de l'homme - trop abstraits des Girondins : subordonnant la liberté au principe de justice, il limite le droit de propriété, devenu relatif (41), et ajoute l'aide sociale («droit au travail » et soutiens aux pauvres). Si un tel programme suppose «l'intervention d'un pouvoir actif et juste»(42), il est - fondamentalement - cautionné par l'Être Suprême, Dieu de Justice et de Fraternité. L. Blanc insiste toujours, à juste titre, sur la dimension religieuse des idées de Robespierre, qui l'élèvent audessus des autres conventionnels. Enfin, après leur victoire sur les Girondins, les Montagnards votent une Constitution qu'il tient pour le «premier pacte social qui eût fondé la liberté sur l'égalité et fait un dogme politique de la fraternité humaine »(43). Mais il regrette, surtout à propos de la nouvelle Déclaration des droits de l'homme, que les idées de Robespierre, qui expriment « la puissance du peuple » (44), n'aient pas complètement triomphé. Christ »)

(39)Id., préambule (Esquiros, dans le même esprit, caractérise la France d'alors comme la « nation

(40) Id, L. X, chap. 1. Dénonçant l'irresponsabilité et l'aventurisme des Girondins, L. Blanc montre que les Jacobins «préparaient les matériaux de la loi avec un ordre, une décence et une discipline dont il n'y avait pas encore eu d'exemple », (id., L. IX, chap. 2).

(41) Pour Robespierre le droit de propriété, d'« absolu et individuel », devient « relatif et social » (id, L. VU, chap. 9 et L. IX, chap. 5). Mais Ph. Buonarotti, tout en réhabilitant Robespierre comme « martyr de l'égalité », regrette ces « désespérantes idées sur le droit de propriété » (La Conspiration pour l'Égalité..., t.1, pp. 25 $s q$. ; malgré tout, il lui reconnaît d'avoir « aspiré à l'égale distribution des charges et des jouissances », p. 88).

(42) Ce pouvoir fort est nécessaire lorsqu'il y a «des faibles à protéger, des pauvres à nourrir, des malheureux à sauver », (id, L. X, chap. 1). Sa conception du Club des Jacobins et du pouvoir lui-même font bien de L. Blanc, héritier de Robespierre, un homme d'ordre (on dénoncera son socialisme autoritaire).

(43)Id.,chap.7.

(44)Id., chap. 3. 
De plus, L. Blanc insiste avec force sur l'opposition constante de Robespierre au développement de la violence politique, pratique qui viole autant les principes sacrés de l'Humanité que les règles élémentaires de la justice (45). D'abord, il condamne, mais insuffisamment à le suivre, les massacres de septembre 1792 (alors que Danton les approuve) (46). Quant au procès du roi, L. Blanc, qui est profondément hostile à la subordination de la justice à la politique, dédouane quasiment Robespierre de son «éloquence meurtrière » contre Louis XVI, en imputant sa responsabilité à Saint-Just (son mauvais génie ?) (47). Enfin, dans le conflit opposant les Girondins aux Montagnards, il dénonce la responsabilité des premiers : initiateurs des proscriptions, qui iront s'aggravant, ils se seraient acharnés à abattre Robespierre dont ils jalousaient le prestige populaire et redoutaient la puissance politique (48). Malgré leurs attaques - injustes et répétées Robespierre, toujours modéré, aurait souhaité résoudre le conflit dans le respect de la loi et de l'ordre, non dans un affrontement meurtrier. Mais c'était sans compter des passions politiques incontrôlées, passions dont luimême n'est pas exempt dans un climat de plus en plus délétère.

$\mathrm{Au}$ moment où les Montagnards l'emportent sur leurs adversaires, Robespierre paraît ainsi incarner parfaitement le rêve révolutionnaire, le projet même de L. Blanc pour la France du XIXe siècle. Une république démocratique et sociale (socialiste ?) faisant régner le Droit et la justice, unissant la nation dans le peuple souverain et mêlant fraternellement toutes les classes selon le dessein de Dieu. Objectif politique et social, éloigné, à la fois, des individualistes bourgeois et des révolutionnaires extrémistes, tous dénoncés comme diviseurs de la communauté nationale. Socialiste, L. Blanc manifeste toujours la plus vive hostilité envers ceux-ci : dénoncés comme «anarchistes» et «faux tribuns», il les montre manipulés et objectivement (?) solidaires tant des individualistes bourgeois que des ennemis de la Révolution. Il en serait ainsi de J. Roux, le leader des Enragés (mais comme l'objectera Michelet, ne fut-il pas, plus que Robespierre, un vrai socialiste ?) (49).

(45) Autre leçon de la Révolution dont les hommes de 1848 tiendront compte en rejetant toute forme de violence politique. L. Blanc, tout au long de son $H R F$, montre la plus grande fermeté sur la question («si vous portez la main sur un innocent parce qu'il vous paraît dangereux, où sera la boussole de l'univers moral ?», se demande-t-il, id., L. V, chap. 7). Mais certains historiens socialistes de la Révolution, ainsi Buchez et Laponneraye, justifient violence et massacres car ils purgent la France de ses ennemis.

(46) Id, L. VIII, chap. 2. L. Blanc lui reproche de ne pas les avoir empêchés.

(47) Cf. id., chap. 2. La condamnation à mort du roi fut une erreur, estime L. Blanc, mais concernant Robespierre il déforme la vérité historique.

(48) Cf. id., chap. 4 et 7, et L. IX, chap. 8 à 11.

(49) Ces condamnations - ces anafhèmes - sont aussi le reflet des rivalités des socialistes d'avant et d'après 1848. Michelet, une fois encore, critique durement L. Blanc, lui reprochant de prendre Robespierre «comme apôtre et symbole du socialisme, qu'il frappait et qui le tua », préface de 1868, op. cit. De plus, L. Blanc reprend telles quelles les accusations formulées par les robespierristes contre les factions qu'ils ont éliminées (accusations annonciatrices des procès politiques du XX' siècle ?). 
Après la victoire montagnarde, en juin 1793, Robespierre allait-il, enfin, accéder au pouvoir politique ? Non, car, une fois encore, L. Blanc soutient qu'il reste cantonné dans son rôle de conscience, voire de guide, de la Révolution : toujours aimé du peuple et orateur hors pair, sa parole élevée, en touchant la raison et les cœurs, inspire toujours une écoute respectueuse dès lors qu'elle fait briller la vérité.

Quant au régime de Terreur que les Montagnards mettent alors en place, L. Blanc souligne qu'il ne fut pas le fruit d'un système, mais des circonstances exceptionnelles où était plongée la France. Si « les injustices du passé l'avaient conçu, [écrit-il] les luttes prodigieuses du présent l'engendrèrent » (50). Thèse qui n'est pas nouvelle, puisque les historiens libéraux de la Révolution ont déjà expliqué les rigueurs de ce régime par les terribles menaces - intérieures et extérieures - auxquelles la France était exposée (51). En revanche, L. Blanc innove en disculpant Robespierre de toute responsabilité : il entend ruiner la légende thermidorienne du tyran, ou du dictateur, qui le flétrit comme responsable des dérives despotiques et sanguinaires de cette époque. Si le régime de Terreur a, effectivement, sauvé la France et la Révolution, ce qu'il n'est donc pas seul à penser, il fut aussi, les circonstances aidant, investi par les passions contrastées des hommes, le bien et le mal. Une fois encore, Robespierre demeure réduit à une parole surtout prophétique, le champ politique lui échappant pour une large part. Décrivant celui-ci, L. Blanc nous le montre partagé entre trois tendances (ou factions) (52) :

- d'une part, il détache les vrais terroristes, hommes soumis à des passions exacerbées par les difficultés et les luttes, qui vont les entraîner dans les pires crimes. De ces «révolutionnaires immoraux » font partie Hébert, Ronsin, Fouché, Collot d'Herbois et Carrier. Ils contrôlent, de fait, l'appareil répressif, Comité de sûreté générale, comités révolutionnaires et Tribunal révolutionnaire (mais de cette justice, dirigée par FouquierTinville, l'« accusateur public», L. Blanc nuance la légende noire, puisqu'elle fut aussi clémente, outre que Robespierre a essayé de limiter les envois devant ce tribunal, voire de sauver des inculpés) (53);

- à l'opposé, il y a ceux qui aspirent à la clémence pour arrêter cette violence, principalement Danton et C. Desmoulins, mais par un excès - contraire - de modération, ils finissent, plus ou moins consciemment, selon L. Blanc, par aider les ennemis de la Révolution ;

(50)Id., L. XI, chap. 1.

(51) Cf. en particulier les Histoire(s) de la Révolution française d'Adolphe THIERS (1823-1828) et François MIGNET (1824). Pour eux, les circonstances, une raison d'État, excusent la Terreur, même si la morale en condamne le principe.

(52) Op. cit., L. XI, chap. 1 et 5.

(53) Cf. aussi, id. chap. 4, le tableau des «proconsuls », les représentants en missions envoyés aux départements et aux années. Fouché, Carrier, Fréron et Barras y sont vivement condamnés pour leurs excès. 
- enfin, occupant une position moyenne, Robespierre et ses rares alliés, Saint-Just et Couthon, défendent une politique de fermeté, pour consolider et sauver la Révolution, mais sans les excès répressifs et criminels qui finissent par la dénaturer gravement. En définitive, Robespierre est favorable à un pouvoir fort assurant ordre et unité, maintenant l'union de tous les révolutionnaires, mais respectant, par-dessus tout, le Droit et les lois morales. Il représente, écrit L. Blanc, le «parti des honnêtes gens » (54).

Mais comme il incarne les principes et les valeurs du Droit, il en impose tant à ceux dont les crimes disqualifient la Révolution qu'à ceux qui, par faiblesse, désertent sa cause. Son objectif, martèle L. Blanc, est de « désarmer» la Terreur, non la Révolution. Au Club des Jacobins et à la Convention, Robespierre recommande donc fermeté et vigilance dans le respect de la justice. Mais les comités de gouvernement, insiste-t-il, lui échappent : outre le Comité de sûreté générale, qui lui est effectivement hostile, il rappelle justement qu'il est entré tardivement au Comité de salut public, mais il exagère son isolement dans ce centre du pouvoir révolutionnaire (même s'il ne le contrôle pas, son influence est plus importante que ne le dit L. Blanc) (55). En définitive, conclut-il, «sa force était dans l'immense autorité morale qui s'attachait à son nom, dans le respect que lui portaient les Jacobins, et dans sa popularité sans égale» (56).

L. Blanc veut ainsi nous montrer qu'il ne cesse de régner par la parole : inspirant un respect mystérieux, sa force - quasi magique - peut toujours retourner ses auditeurs, car la vérité qu'elle exprime dissipe autant erreurs et ignorances qu'elle impose le silence aux passions impures. En fait, L. Blanc montre bien que Robespierre s'est tôt imposé par sa rigueur intellectuelle et sa cohérence politique (57). L'homme des principes aurait-il surtout exercé une dictature d'opinion? Certainement, si l'on prend en compte son réel ascendant, un leadership de fait, mais aussi un caractère réservé et distant, voire hautain. Autant d'éléments qui le font considérer, dans le pays et à l'étranger, comme le véritable responsable de la France. Mais L. Blanc, on le voit, va bien au-delà. Exagérant son peu de pouvoir politique, et insistant beaucoup sur sa dimension religieuse, fort contestée au demeurant, il en fait donc un vrai prophète, interprété selon un prisme chrétien. Mais comme sa parole de vérité n'est pas assez entendue, elle reste comme suspendue, voire abstraite. Elle est en attente des générations futures - plus morales et plus conscientes - qui sauront mieux l'entendre : car à elles reviendra d'accomplir la Révolution, dès lors que sa vérité se sera - enfin - imposée et le mal

(54) Id, chap. 12.

(55) Id., chap. 7 et L. XI, chap. 5. Michelet dément L. Blanc car Robespierre, soutient-il, avait tout le pouvoir («au Comité de salut public il ne paraissait pas, faisait signer ses actes par ses collègues... ainsi il lui était loisible de se laver les mains de tout », préface de 1868, op. cit.).

(56) Op. cit., L. X, chap. 7.

(57) Mirabeau l'avait déjà remarqué : «Il ira loin parce qu'il croit ce qu'il dit ». 
dissipé. Le personnage de Robespierre cristallise donc tout le mystère de la Révolution, événement grandiose et sublime dont le vrai sens reste - encore - obscur, en ces années-là, pour une majorité de Français. C'est pour ces raisons que L. Blanc donne une si grande importance à l'instauration du culte de l'Être Suprême. Destinée à éclairer les consciences et à y renforcer le sens moral, pour établir un ordre authentique, cette religion civile, préconisée par Rousseau, fut comme un moment de grâce, un éclat surnaturel. Car l'évidence de la vérité frappa, un temps, les esprits. On comprit qu'il fallait consolider la Révolution dans le respect du Droit et de la justice, sa vérité même. C'est bien parce que la nouvelle religion devait purger la Révolution de ses crimes et des passions impures qui finissaient par la compromettre, qu'elle fut si bien, et si spontanément, accueillie. À suivre L. Blanc, tant le discours de Robespierre à la Convention que la fête elle-même furent un vrai moment de fraternité et de liesse collectives, dont il recueillit toute la popularité («à ce langage, écho d'une conviction héroïque, l'Assemblée se sentit invinciblement émue, elle se leva toute entière ») (58). Mais cet événement, par la loi de fatalité, fut pourtant sans lendemain.

Dans le même esprit, Robespierre, insiste L. Blanc, continue de s'opposer aux pratiques terroristes. Il fait ressortir l'action bénéfique et morale des «proconsuls», les représentants en mission dans les départements et aux armées qui se réclament de lui, Saint-Just et Lebas (59). Plus encore, L. Blanc le disculpe des violences politiques qui ont déchiré les Montagnards. À commencer par le procès et l'exécution des Hébertistes : Robespierre, note-t-il, était alors absent et malade. Pourtant, cette nouvelle parodie de procès ne l'empêche pas d'approuver l'élimination d'une faction extrémiste symbolisant, à elle seule, tout ce qu'il condamne (et qui dénature l'esprit même de la Révolution) : l'anarchie sociale et intellectuelle, corollaire de l'individualisme, et l'athéisme, qui en serait le fondement (60). En revanche, L. Blanc est plus circonspect sur le procès des Dantonistes. Malgré les ambiguïtés personnelles de Danton et les dangers de sa politique de modération, il reproche à Robespierre de ne s'être pas assez opposé à un procès dont il condamnait le principe. Une fois encore, il aurait cédé au fanatisme de Saint-Just (qui reste son mauvais génie ?), même s'il a tenté de sauver C. Desmoulins, son ancien condisciple (61). Critique qui n'est pas isolée : toujours très sensible aux problèmes de justice, surtout

(58) Op. cit., L. XI, chap. 11

(59) Id., chap. 4 (cf. note 53).

(60) Cf. id., L. X, chap. 14 et I.XI, chap. 9. Comme Robespierre, L. Blanc montre toujours la plus grande hostilité aux révolutionnaires extrémistes (Enragés de J. Roux et Hébertistes, reprenant même contre ceux-ci l'accusation de complot). Il dénonce particulièrement leur athéisme, lui opposant la « doctrine républicaine de l'unité et de la fraternité ».

(61)Id.,L. XI, chap. 10. 
politique, L. Blanc lui reproche d'avoir inspiré la loi du 22 prairial, qui réduit les garanties des accusés du Tribunal révolutionnaire (même si son objectif était de punir les crimes et les excès des terroristes !) (62). Même prophète, Robespierre n'est pas sauf des faiblesses humaines (à son insuffisante fermeté à empêcher les procès iniques, L. Blanc ajoute sa propension aux « accusations exagérées » contre ses adversaires) (63).

Mais, en définitive, l'autorité de Robespierre, même limitée par L. Blanc à un magistère de la parole, n'aura-t-elle été que morale et, par conséquent, peu politique ? Du pouvoir n'aurait-il eu, dès lors, que les apparences? Question d'importance car la réponse conditionne son statut - tyran ou prophète - dans le débat politique contemporain. Mais L. Blanc, qui en élevant Robespierre à la dimension de prophète veut le disculper des excès de la Terreur, n'est pas assez précis sur son rôle réel dans le «mécanisme révolutionnaire » de l'an II, dont il vante tant l'unité et l'efficience (64). Son autorité morale, voire sa dictature d'opinion, l'aurait-elle réduit à n'être que la force d'impulsion, - pour partie irresponsable - du système, puisqu'il n'y contrôlait que le Club des Jacobins, sorte de parti-guide, et la Commune de Paris, le centre populaire de la Révolution? C'est ce que suggère L. Blanc : même si la parole de Robespierre est toujours en mesure d' influencer la Convention, il ne cesse de souligner qu'il reste minoritaire, et peu écouté, dans les vrais centres du pouvoir, là où s'élaborent les pratiques terroristes (Comités de gouvernement, couronnement du «mécanisme révolutionnaire », voire Tribunal révolutionnaire). Thèse vivement combattue, en particulier par E. Quinet et, surtout, Michelet qui entend montrer que Robespierre, même sans responsabilité politique apparente, s'est servi de l'appareil jacobin, qu'il contrôle effectivement, pour fonder une vraie tyrannie politique, la cause même des dérives despotiques de l'an II (65).

\section{La tragédie sacrificielle de Thermidor : Passion de Robespierre}

Exalté par L. Blanc comme la conscience et le guide de la Révolution, et y exerçant peu le pouvoir politique, Robespierre va pourtant tomber, victime d'un complot qui lui sera fatal. Paradoxalement, ses nombreux adversaires, en grande majorité aux Comités de gouvernement, exploitent

(62) Id, chap. 12.

(63) Cf. id., L. VIII, chap. 7 et L. IX, chap. 5.

(64) Id., L. X, chap. 7. Ce «mécanisme révolutionnaire [écrit L. Blanc] était conçu de façon à imprimer une force et une unité irrésistibles à l'action de Paris, considéré comme le brûlant foyer des idées nouvelles, comme le point d'où la France devait prendre son élan »; mais il ajoute que « la France ne se manqua pas à elle-même ».

(65) Cf. son Histoire de la Révolution française, par exemple L. IX, chap. 3 et 4 (le Club des Jacobins préfigurerait-il les Partis communistes du XXe siècle ?). Cf. aussi L. XIV, chap. 5, sur « la papauté de Robespierre ». 
l'autorité morale et le prestige du prophète pour le rendre « seul responsable des actes du pouvoir ». L. Blanc dénonce avec force cette injustice de l'avoir transformé en tyran à abattre pour faire cesser une Terreur de plus en plus impopulaire. Alors même qu'il veut mettre fin à ses excès, on «concentre sur sa tête la responsabilité des malheurs publics» (66). «Tant d'injustice l'accabla, [écrit-il] il sentit son cœur flétri et se crut réduit à l'impuissance de faire le bien et d'arrêter le mal » (67).

Or, dans son discours du 8 thermidor, Robespierre, insiste L. Blanc, continue de défendre le même programme : union nécessaire des vrais républicains pour instaurer liberté, justice et sécurité ; fermeté pour sauver une Révolution toujours en danger; arrêt des excès et des injustices de la Terreur (n'a-t-elle pas redoublé lorsque, par amertume, il s'est retiré quelque temps du Comité de salut public?) (68). Se sentant menacés, les terroristes, qu'il dénonce depuis longtemps, organisent un complot élargi à de vrais modérés. L. Blanc le décrit comme une coalition hétéroclite où ressentiments personnels, calculs machiavéliques et desseins inavoués entrent largement en jeu. Mais Robespierre ne se départit pas de son rôle en restant au-dessus d'une obscure politique. Aux ténèbres du mal il oppose, comme il se doit, la pleine lumière du bien : «pendant que, de leur propre aveu, les ennemis de Robespierre faisaient entrer dans leur chance de succès la dissimulation, l'hypocrisie et l'assassinat, lui le tyran [écrit-il] repoussait l'emploi de la violence, allait s'inspirer à l'Ermitage du souvenir de cet infortuné Jean-Jacques, et ne comptant, pour son triomphe, que sur la raison, se préparait au combat en s'armant d'un discours !» (69). Dans la tragédie qui s'annonce, Robespierre va puiser des forces dans le souvenir de Rousseau, autre victime de l'incompréhension et de l'injustice des hommes. Est-ce encore un mystère de l'Histoire que le malheur se soit abattu sur ces deux maîtres de lumière et de vérité ?

Mais Robespierre pouvait-il l'emporter, s'interroge L. Blanc ? Croyant toujours au rôle de la fatalité, la logique même de l'Histoire, il explique le complot thermidorien par des causes plus profondes que celles d'une vulgaire intrigue. Il n'en serait que l'épiphénomène. De fait, il est convaincu qu'après tant d'épreuves, de luttes et d'énergie déployées par les Français, le paroxysme révolutionnaire doit nécessairement retomber, car telle est la loi mystérieuse du drame. Or la lassitude de la Révolution doit - inévitablement - entraîner le sacrifice de celui qui en incarne au plus haut l'esprit : Robespierre. Comme l'écrit, avec force, L. Blanc, « quand la

(66) Op. cit., L. XII, chap. 2.

(67)Id, L. XI, chap. 12. Michelet dément encore L. Blanc : «dans son isolement, dans son inertie apparente, (Robespierre) n'en tenait pas moins un procès et sur les grands hommes d'affaires du Comité et sur les 200 Montagnards qui avaient enduré tout... », (préface de 1868, op. cit.).

(68) Op. cit., L. XII, chap. 4 et 5.

(69)Id,chap.5. 
France eut la lassitude de son héroïsme, il fallut renverser la Révolution elle-même »(70). Donc mettre à mort son prophète et son apôtre qui devient, dès lors, selon ses propres termes, martyr et bouc émissaire : le «système qui consistait à concentrer sur lui toutes les haines» (71) emportera donc l'esprit même de la Révolution... Guidé par l'instinct supérieur des voyants, Robespierre lui-même sait que «son heure était venue ». Caractérisant comme «testament de mort» son discours du 8 thermidor, celui qui n'avait pas été assez écouté ne s'adresse plus qu'aux générations futures qui, tel L. Blanc, reprendront la lutte pour accomplir le message des deux apôtres du XVIII ${ }^{\mathrm{e}}$ siècle. Sa mémoire, maintenant, lui importe plus que sa vie.

Racontant la fin de Robespierre, L. Blanc nous décrit la Passion d'un nouveau Christ: le Christ de la Révolution. Le 9 thermidor, les comploteurs, après avoir écouté, à la Convention, Saint-Just, empêchent le prophète de parler. Ils craignent que sa parole, une fois encore, retourne une majorité qui semble manipulée, beaucoup ressentant même, au dire de L. Blanc, honte et stupeur à sa mise en accusation (72). Bien que perdu, Robespierre reste fidèle à son message de toujours en refusant de riposter par la violence d'une insurrection. Pourtant, L. Blanc pense qu'elle était possible : la Commune de Paris, qui lance, malgré tout, un appel à l'insurrection pour le sauver, les Jacobins et la moitié des sections de la capitale auraient fourni les forces nécessaires pour retourner la situation. Affirmation de principe, sans doute, car des historiens, Michelet d'abord, le contredisent, faits à l'appui, en soulignant l'isolement complet de Robespierre, même dans les milieux populaires (73). L. Blanc explique qu'il a refusé de résister pour ne pas attenter aux principes les plus sacrés de la Révolution, le Droit et la Souveraineté du Peuple, perspective qui l'aurait «profondément troublé jusqu'au fond de l'âme ». De fait, par respect de la légalité - la représentation nationale - il n'a pas pris la tête de l'insurrection, mais ne l'a pas non plus désavouée. Pourtant Robespierre va subir l'échec d'une tentative qu'il n'a pas souhaitée, d'une illégalité qu'il ne voulait pas. Comme le prophète ne peut se renier, il lui faut donc accepter la mort, puisque tant la raison que le sentiment sont désarmés face à la force brutale

(70)Id., L. VIII, chap. 1.

(71)Id., L. XI, chap. 12. Pour Laponneraye, qui évoque les «martyrs de Thermidor», Jésus et Robespierre sont tous deux morts du «supplice des infâmes » (Mémoires de M. Robespierre, t. I, p. 5). Même approche, mais dans une moindre mesure, chez Esquiros.

(72)Id., L. XII, chap. 7.

(73) Cf. son Histoire de la Révolution française, L. XXI, et sa préface de 1868. Michelet souligne le complet isolement de Robespierre à la Convention (Montagnards et modérés se liguent contre lui). De plus, si la Commune et les Jacobins appuient Robespierre, Michelet, archives à l'appui, montre la faible mobilisation des sections en sa faveur (16 seulement, sur 48 , répondent à l'appel insurrectionnel de la Commune). Paris et le peuple l'ont abandonné (mais n'a-t-il pas, insiste Michelet, frappé les premiers socialistes, comme J. Roux ?). 
et sournoise déployée par des ennemis qui, eux, renient la Révolution. L'apôtre va donc vivre une Passion reproduisant, fait après fait, celle du Nazaréen, répétition nécessaire pour que la leçon soit identique. Il n'est jusqu'au coup de feu du gendarme Meda sur Robespierre, tiré lors de la prise de l'Hôtel-de-Ville, centre de l'insurrection, pour rappeler le coup de lance du soldat romain sur Jésus. Puis L. Blanc insiste sur la longue «agonie» de Robespierre, supportée avec tout le stoïcisme du Sage, lorsqu'il est ramené, blessé, à la Convention. Cette souffrance et ces nouveaux outrages - son chemin de Croix ? - conduisent L. Blanc à se demander s'il «s'interrogea sur la loi, effroyablement mystérieuse, qui, depuis l'origine du monde, couronne les artisans de l'iniquité et ne réserve que tortures aux serviteurs de la Justice »(74). Vient enfin l'exécution, qui conclut ses souffrances. Sa montée à l'échafaud (nouveau Golgotha ?) n'est, en fait, qu'une mise en Croix : nouveau Jésus-Christ, il meurt en crucifié, ainsi que l'écrit L. Blanc. Avant d'expirer, il pousse un cri, comme le Sauveur encore, cri que L. Blanc interprète comme la mort symbolique du peuple («cri de ce pauvre peuple, cri de millions d'infortunés ») (75). Mais contrairement aux temps évangéliques, le peuple ne demande plus la mort du nouveau Jésus pour sauver un autre Barabbas, car il est - encorepolitiquement passif. Les nouveaux Caïphe sont donc seuls à engager un sacrifice qui, cette fois, n'est pas un meurtre collectif, même si nulle force ne vient les en empêcher (76).

Si le bouc émissaire de l'Ancien Testament emporte dans son sacrifice «toutes les fautes, tous les péchés» d'Israël (77), le crucifié, selon le Nouveau Testament, livre sa vie en vue du Salut des hommes (78). Dans le premier cas, la Révolution serait close dans l'injustice, mais dans le second elle s'ouvre à l'espérance, puisque la mort de Robespierre assumerait symboliquement le mal qui l'a dénaturée pour l'abolir. À charge pour les générations futures, qui devront comprendre le sens de sa mort innocente, de reprendre son message prophétique pour en réaliser tout le bien. Comme la parole du Christ, elle fournit les moyens d'échapper à la fatalité du mal. Les souffrances et le sang versé de Robespierre eurent-ils cet effet rédempteur? Sans le dire explicitement, L. Blanc le pense certainement.

(74) Op. cit., L. XII, chap. 7.

(75) Id., L. Blanc ajoute que « les artisans et les ouvriers n'étaient pas là » (non par deuil politique, préciserait Michelet, mais parce qu'il avait réprimé ses leaders).

(76) À l'inquiétude des prêtres et des pharisiens - « cet homme accomplit beaucoup de signes, si nous le laissons faire tous croiront en lui »- le grand prêtre Caïphe répond : « vous n'y entendez rien, vous ne voyez pas qu'il vaut mieux qu'un seul homme meure pour le peuple et que la nation ne périsse pas tout entière »(Jean, 11/47-53).

(77) Cf. Lévitique, 16/21. Napoléon pense aussi que Robespierre fut le bouc émissaire de la Révolution (cf. E. DE LAS CASES, Mémorial de Sainte-Hélène, éd. Jean Tulard, Paris 1968,1.1, p. 252).

(78) Cf., en particulier, les propos du Christ lors de la dernière Cène : « ceci est mon corps donné pour vous, cette coupe est la nouvelle Alliance en mon sang versé pour vous » (Luc, 22/19-20). 
Enfin, «comme la chute de l'homme et de l'idée ne firent qu'un», conclut-il, la réaction peut commencer : la fin de la fraternité - pourtant incomplètement réalisée - entraîne le retour à l' individualisme, règne exclusif de la bourgeoisie sur le peuple. Les hommes de l'an II, même les terroristes, redeviennent bourgeois, nouveaux modérés parmi les plus anciens. Preuve que le régime montagnard lui-même n'a pas su accomplir la Révolution jusqu'à son terme logique. Preuve encore que Robespierre fut surtout son prophète. Mais, avant de mourir, il a transmis son message de vérité aux générations futures, en leur confiant la responsabilité de l'accomplir : « la Révolution [conclut L. Blanc] nous a laissé en héritage un sol, indéfiniment fertile, à agrandir » (79).

De Robespierre, L. Blanc a ainsi construit une image de prophète attaché à prêcher la vérité de la Révolution, sa finalité même : la fusion de l'idéal républicain et de l'utopie socialiste (au demeurant peu définie). Enrichissant cette perspective d'une dimension chrétienne, il a campé Robespierre, après Rousseau, en nouveau Jésus. L'Incorruptible, dès lors, incarne un jacobinisme socialiste et religieux, voire catholique, prisme où se réfractent les dimensions sociale et morale de l'Humanité. Mais comme le progrès -mystère de l'Histoire - s'accomplit toujours lentement et difficilement, 1793 s'est achevé sur une tragédie qui a mis un terme à une espérance sublime : la fraternité des classes dans l'unité démocratique. Le verbe du prophète s'est montré d'autant plus impuissant à conjurer les forces du mal que les conditions objectives, les dispositions des hommes surtout, estime L. Blanc, l'ont empêché d'être aussi un vrai responsable politique. Des progrès nécessaires ont été ainsi contrariés, laissant la Révolution - pourtant dynamique et exemplaire - inachevée. La violence politique de la Terreur, en particulier, a gravement violé le principe même de l'Humanité. En frappant injustement Robespierre, qui la condamnait, elle a fait du prophète la victime sacrificielle de forces qui, du même coup, ont renoncé à achever l'œuvre de Révolution. Mais le christianisme, en aidant à comprendre ses contradictions, permet à L. Blanc de maintenir l'espérance : outre que l'exécution du bouc émissaire a été, en fait, une mort rédemptrice, le message de vérité de Robespierre, prophète laïc, finira par s'imposer aux générations futures, plus éclairées et plus morales. Le mystère de la Révolution - de l'Histoire en fait - se dissipera lorsqu'elles en comprendront tout le sens : la parole prophétique deviendra alors une réalité sociale, morale et politique. Comme l'écrit Esquiros, en 1847, la «mémoire des Montagnards est la colonne de feu qui guide les générations errantes et indécises à la recherche d'une nouvelle terre promise » (80).

(79) Op. cit.,L.XVI,chap.4.

(80) Dans son Histoire des Montagnards, t. II, p. 470 (il écrit aussi que « la Montagne était le Sinaï de la loi nouvelle », t.1, p. 77). 
L'approche de L. Blanc, son analyse de Robespierre, s'inscrit donc dans ce courant radical et socialiste de la monarchie de Juillet qui prépare la révolution de 1848, et survivra à son échec. Transfigurant 1793 dans le même moule d'idéalisme, de messianisme religieux et de mystique du peuple - dans sa double acception sociale et politique - il exalte, sanctifie même, Robespierre contre la légende noire de Thermidor. Car de l'Incorruptible celle-ci donne une image tout à fait opposée. Ni saint ni prophète, il aurait été, en fait, le vrai maître du pouvoir : un tyran, ou un dictateur. C'est ainsi que Quinet et, surtout, Michelet contredisent L. Blanc. Dénonçant 1793 au nom de 1789, ils lui opposent que Robespierre, en fait, a interrompu le règne du Droit en privant le peuple de sa jeune liberté. Mais leurs analyses de son pouvoir divergent quelque peu : restaurateur d'un despotisme d'Ancien Régime, aux yeux du premier, Robespierre, pour le second, ne serait ni Jésus ni l'initiateur du socialisme (qu'il a réprimé), simplement le premier des tyrans modernes. De fait, Michelet l'accuse d'avoir manipulé le Salut Public lorsqu'il a transformé le Club des Jacobins en appareil de contrôle et de coercition, instaurant ainsi un vrai système de Terreur (annonciateur pour certains des totalitarismes contemporains). Or, dans le contexte des déceptions et des désillusions de l'après 1848, les critiques républicaines de Michelet et Quinet contribuent à mettre en question la version jacobine-socialiste, et religieuse, de la Révolution.

C'est ainsi que dans les années 1860 l'approche de L. Blanc est de plus en plus contestée par les nouvelles générations républicaine et, même, socialiste (où il faut compter de nombreux futurs communards). Beaucoup sont hostiles au jacobinisme autoritaire des «séminaristes rouges», dont Robespierre aurait été le maître d'oeuvre en 1793 (81). Dans cet échange constant - et toujours complexe - entre passé et présent, critiques de L. Blanc et de Robespierre vont de pair, toutes deux provoquées par les mises en cause parallèles de 1793 et 1848, pourtant dissemblables. Le tyran l'emporte à nouveau sur le saint et le prophète : alors que le jeune républicain J. Ferry, influencé par Michelet et Quinet, défend aussi la libération thermidorienne (82), les socialistes Proudhon et Blanqui, chefs de plus en

(81) Dans la préface de la réédition, en 1868, de son Histoire de la Révolution française, Michelet, on l'a vu, s'en prend vivement à L. Blanc : outre son «papisme socialiste » et son rousseauisme, il dénonce sa rhétorique chrétienne et sa déification de Robespierre («nouveau Jésus »). Le journal libéral (et protestant) Le Temps reproduit cette préface en l'accompagnant d'une réponse de L. Blanc ; une polémique s'ensuit entre les «champions des deux "Églises" séparées de la République»(P. Viallaneix), chacun maintenant ses positions (octobre 1868). Dans une nouvelle édition, en 1869, de son Histoire Michelet accentue encore sa critique de L. Blanc (et de Robespierre) en introduisant le tome IV d'une préface particulièrement explicite : Le tyran. Sur ces débats cf. François. FURET, La gauche et la Révolution au milieu du XIX' siècle (Paris, 1986).

(82) J. Ferry soutient La Révolution française de Quinet, lors de sa réédition de 1865, dans plusieurs articles du même journal Le Temps. Autre polémique inutile : à la demande de la rédaction elle-même, L. Blanc lui répond, maintenant toujours l'essentiel de ses positions (1866). 
plus écoutés - même s'ils s'opposent - s'en prennent, eux aussi, à Robespierre, lui préférant même Hébert (83)! Ce large rejet de Robespierre par les républicains et une majorité de socialistes signe-t-il aussi l'échec de L. Blanc? Toutefois, au début du XXe siècle, Robespierre sera réintégré dans le champ du socialisme et de la République grâce, surtout, à J. Jaurès. Se réclamant aussi de la Révolution et d'un socialisme uni à la démocratie, Jaurès, homme de synthèse, défend un idéal de justice et de progrès. Son humanisme conciliateur n'est pas éloigné de L. Blanc, même s'il récuse sa dimension chrétienne (84). Renouant avec Babeuf, il réhabilite Robespierre selon Buonarotti : parce qu'il a incarné, en l'an II, une démocratie politique et sociale, qui fut aussi morale, l'Incorruptible peut prendre place dans l'histoire du socialisme humaniste qu'il veut incarner (85). Toujours proche en celà de L. Blanc, J. Jaurès ne dépouille pas moins Robespierre de toute aura prophétique, héritage - maintenantdépassé de l'esprit de 1848. Il ne s'agit donc plus de recueillir la parole du prophète pour l'accomplir, mais de tracer une voie différente au service, certes, du nouveau prolétariat, mais, d'abord, de «l'homme tout entier» (86). Perspective que L. Blanc n'eût pas désavouée.

\section{Jean-François JACOUTY \\ Université Paul-Valéry Montpellier III \\ 9, rue Saint-Romain \\ 75006 Paris}

(83) Blanqui préface ainsi Les Hébertistes, plainte contre une calomnie de l'histoire d'Edmé TRIDON (1864). Or L. Blanc, on l'a vu, a vigoureusement dénoncé tant Yathéisme que l'individualisme d' Hébert..

(84) De fait Jaurès critique le Robespierre religieux et sa fête de l'Être Suprême (cf. son Histoire socialiste de la Révolution française, 1901-1904, Paris rééd. 1972, t. VI, ch. VI). Mais Albert Mathiez, qui réhabilite aussi Robespierre, relativise sa croyance religieuse, soutenant même qu'il n'en est pas l'auteur (cf. «Le culte de l'Être Suprême » dans Études sur Robespierre, Paris, rééd. 1958).

(85) Jaurès écrit clairement que «nous sommes au sens où l'entendait Babeuf le parti de la démocratie et de la Révolution » (pp. cit., p. 516).

(86) Ainsi que l'écrit Jaurès dans un article paru dans La Petite République du 13 mai 1893. 